\title{
Inelastic electron tunneling across magnetically active interfaces in cuprate and manganite heterostructures modified by electromigration processes
}

\author{
M. A. Belogolovskii ${ }^{1}$, Yu. F. Revenko ${ }^{1}$, A. Yu. Gerasimenko ${ }^{1}$, \\ V. M. Svistunov ${ }^{1,2,3}$, E. Hatta ${ }^{2}$, G. Plitnik ${ }^{3}$, V. E. Shaternik ${ }^{4}$, \\ and E. M. Rudenko 4 \\ ${ }^{1}$ A. Galkin Donetsk Physical and Technical Institute of the National Academy \\ of Sciences of Ukraine, 72 R. Luxemburg Str., Donetsk 83114, Ukraine \\ E-mail: vlamis@hsts.fti.ac.donetsk.ua \\ ${ }^{2}$ Nanoelectronics Lab., Faculty of Engineering, Hokkaido University, Sapporo 060, Japan \\ ${ }^{3}$ Frostburg State University, Frostburg, MD 21532, USA \\ ${ }^{4}$ Institute for Metal Physics of the National Academy of Sciences of Ukraine \\ 36 Vernadsky Blvd., Kiev 03142, Ukraine
}

Received February 11, 2002

\begin{abstract}
We report a study of the electron tunneling transport in point-contact junctions formed by a sharp Ag tip and two different highly correlated oxides, namely, a magnetoresistive manganite $\mathrm{La}_{0.66} \mathrm{Ca}_{0.34} \mathrm{MnO}_{3}$ and a superconducting cuprate $\mathrm{LaBa}_{2} \mathrm{Cu}_{3} \mathrm{O}_{7-x}$. Strong chemical modifications of the oxide surface (supposedly, oxygen ion displacements) caused by applying high voltages to the junctions have been observed. This effect is believed to be responsible for an enormous growth of inelastic tunneling processes across a transition region that reveals itself in an overall «V»-shaped conductance background, with a strong temperature impact. The mechanism of the inelastic scattering is ascribed to charge transmission across magnetically active interfaces between two electrodes forming the junction. To support the latter statement, we have fabricated planar junctions between $\mathrm{Cr}$ and $\mathrm{Ag}$ films with an antiferromagnetic chromium oxide $\mathrm{Cr}_{2} \mathrm{O}_{3}$ as a potential barrier and at high-bias voltages have found an identical conductance trend with a similar temperature effect.
\end{abstract}

PACS: 75.70.Cn, 72.10.Di, 73.40.-c

In the last few years, and mainly due to the progress in nanotechnology, the rapidly emerging field of spin-polarized transport across heterostructures, often called «spintronics», has become an area of intensive basic and applied studies. Usually, an analysis of spintronic systems ignores interfacial processes which can flip the spin of a conduction electron travelling across a device. But their influence on the magnetic junction transport characteristics may be dramatic and it would be important to study the sensitivity of tunneling transport properties on the magnetic interfacial scattering. This problem was addressed theoretically [1] and experimentally (see [2] and references therein) in relation to magnetic tunnel junctions showing large magnetoresistance. As it was argued by Guinea [1], the effect should be particularly enhanced in fully polarized magnets as doped manganites where the tendency towards ferromagnetism may be reduced at a surface, leading to an antiferromagnetic behavior. Thus to study an effect of spin-flip processes caused by interface magnetic excitations on the charge transport in such systems seems to be one of the actual issues.

In this sense, ferromagnets as junction electrodes are not good candidates for investigating the role of the spin-assisted tunneling and simpler devices would be preferable. In our previous paper [3] we

(c) M. A. Belogolovskii, Yu. F. Revenko, A. Yu. Gerasimenko, V. M. Svistunov, E. Hatta, G. Plitnik, V. E. Shaternik, and E. M.Rudenko, 2002 
presented tunneling measurements on hightemperature superconducting cuprates, another family of perovskites. It was shown that in these materials interactions of tunneling electrons with excitations inside the transitional insulating layer strongly influence the conductance spectra. The system investigated experimentally [3] was a point-contact junction prepared with a sharp Ag tip pressed into the surface of a ceramic superconductor $\mathrm{LaBa}_{2} \mathrm{Cu}_{3} \mathrm{O}_{7-x}$ ( $\left.\mathrm{LBCO}\right)$. The latter one is known to be one of the most unstable materials among superconducting high- $T_{\mathrm{c}}$ cuprates, with a bad degraded layer adjoining the surface. Our interpretation of the data obtained was based on an assumption that oxygen rearrangement caused by an applied electric field results in the appearance of an oxygen-deficient region near the interface. In accordance with the 123-compound phase diagram it should be antiferromagnetically ordered, and the related inelastic-scattering processes can strongly contribute to the charge transmission across the junction. In this work, we demonstrate important similarities between background conductance spectra of junctions formed by manganites and cuprates as well as an effect of high voltage biases on them; experimentally demonstrate signs of near-interface chemical modifications (supposedly, oxygen ion displacements that we believe to be a source of the composition changes), and confirm the hypothesis of the existence and strong effect of magnetic correlations in the transition region between Ag and lanthanum-based metaloxides by performing the same measurements on a tunnel junction with a $\mathrm{Cr}_{2} \mathrm{O}_{3}$ barrier known to be antiferromagnetic in the bulk state.

We start with conductance experiments on the magnetoresistive compound $\mathrm{La}_{0.66} \mathrm{Ca}_{0.34} \mathrm{MnO}_{3}$ (LCMO). Bulk samples of manganites (as well as cuprates) were sintered by the conventional solid-state method. Appropriate amounts of corresponding oxides were mixed, pressed, and annealed at $1200^{\circ} \mathrm{C}$ for 12 hours. The resulting pellet was ground, sintered, and repelletized before a sample with a high packing density was obtained. The values of the Curie temperature agreed well with known data (the inset in Fig.1,a). Point-contact junctions were prepared with a silver counterelectrode by the same way as those based on high- $T_{c}$ LBCO compounds (for details see the paper [3]). The corresponding peak in the temperature dependence of the tunnel resistance for LCMO was always shifted to lower $T$ as a result of the suppression of ferromagnetism in the upper layers of the manganites (the inset in Fig.1,a). The related
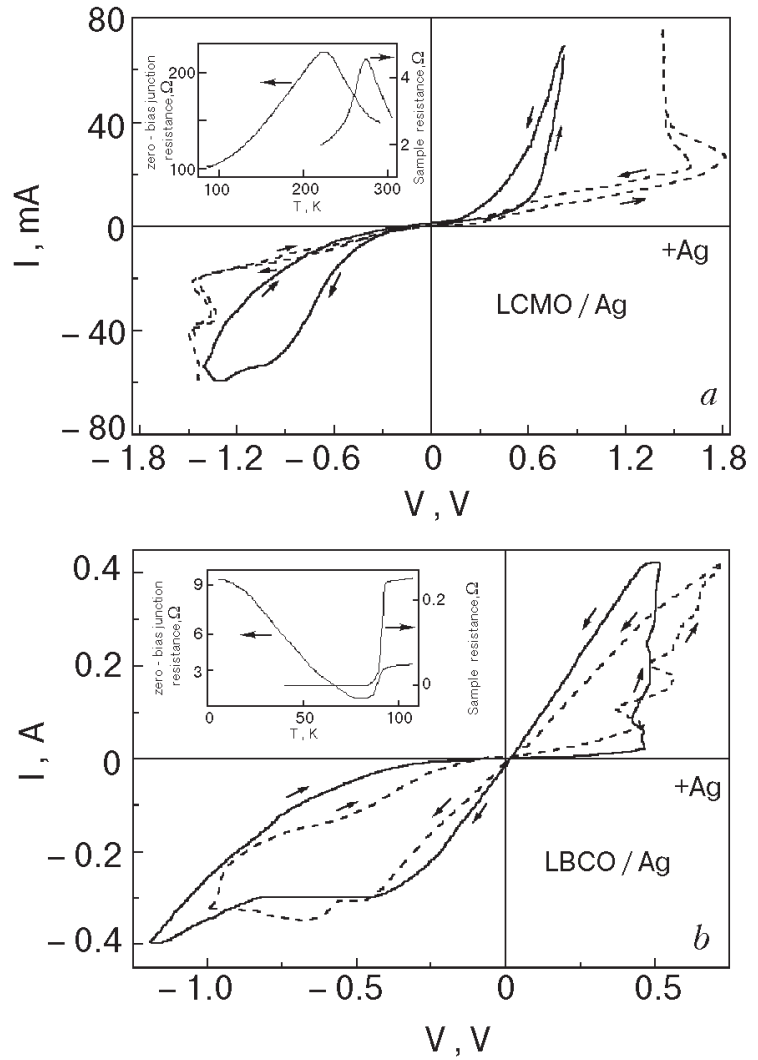

Fig.1. Switching effect on the current-voltage characteristics of $\mathrm{La}_{0.66} \mathrm{Ca}_{0.34} \mathrm{MnO}_{3} / \mathrm{Ag}(a)$ and $\mathrm{LaBa}_{2} \mathrm{Cu}_{3} \mathrm{O}_{7-x} / \mathrm{Ag}$ (b) point contacts at $300 \mathrm{~K}$ (solid lines) and $77 \mathrm{~K}$ (dashed lines). The insets display the temperature behavior of the zero-bias junction resistance compared with that for a bulk metaloxide sample.

findings for superconducting LBCO contacts with $T_{c}$ 's near $93 \mathrm{~K}$ are presented in the inset in Fig.1, $b$. Together with the overall increase expected from the conventional tunneling theory [4] the junction resistance also displays a minimum below $T_{c}$. As was shown in the paper [5], such a feature appears in conductance spectra of $\mathrm{N}-\mathrm{I}-\mathrm{S}$ heterostructures for moderate values of the insulating region transparency (the standard theory of tunneling processes [4] assumes the presence of very high potential barriers).

A radical (but reversible) effect of oxygen electromigration processes on the $\mathrm{LCMO} / \mathrm{Ag}$ contact current $I$-voltage $V$ curves is shown in Fig. 1, $a$ in comparison with corresponding data for $\mathrm{LBCO} / \mathrm{Ag}$ junctions in Fig.1, $b$. In both cases at $300 \mathrm{~K}$ we observed a decrease of the point contact resistance when a positive bias voltage $V^{*}$ was applied on the Ag tip and an increase for the corresponding negative biases. The values of $V^{*}$ were different: near $0.8 \mathrm{~V}$ for LCMO and about $0.5 \mathrm{~V}$ for LBCO. Following previous works on the same subject $[6,7]$, we explain abrupt changes of the point-contact re- 
sistance at activation voltages by modifications of the oxygen stoichiometry near the intrinsic metaloxide surface under the influence of applied electric field. Then the difference in $V^{*}$ can be ascribed to the fact that in the lanthanum-based cuprate the $\mathrm{Cu}-\mathrm{O}$ bond is weaker than the $\mathrm{Mn}-\mathrm{O}$ bond in LCMO. It should be noted that for a parent yttrium-based cuprate (YBCO), where the $\mathrm{Cu}-\mathrm{O}$ bond is known to be stronger than in LSCO, the bias range where the electromigration effect can be detected is, as a rule, essentially larger [7]. The picture proposed explains also why the activation voltages $V^{*}$ needed to stimulate the transition from one branch of the conductance spectra to another have increased with decreasing temperature (Fig.1).

Our results show that after applying high voltages of different polarities not only are the conductance values different but the character of the spectra as well is changed from a parabolic characteristic (the high-resistance characteristic) to a «V»-shaped behavior in the low-resistance case. Figures $2, a-c$ show typical examples of differential conductance curves $\sigma(V)=d I(V) \quad d V$, each at a different temperature. It should be emphasized that there is a fundamental difference with conventional metal-insulator-metal junctions, where the background behavior is polynomial for voltages small compared with the barrier height [4]:

$$
\sigma(V)=\alpha+\beta V+\gamma V^{2} .
$$

In manganite- and cuprate-based contacts the overall dependence of $\sigma(V)$ does not have the form (1) but rather follows the formula [7]

$$
\sigma(V)=a+b|V|+c V^{2} .
$$

To demonstrate it, in the insets in Figs. $2, a-c$ we have plotted the even conductance $\sigma(V)=[\sigma(V)+$ $+\sigma(-V)] / 2$ for the lowest temperatures studied. It does contain a dominating linear term.

It has been already stressed that the $\ll \mathrm{V} »$-shaped background is a common feature of the conductance spectra $\sigma(V)$ of different metallic oxide systems [8]. Here we present some novel results for $\mathrm{LBCO} / \mathrm{Ag}$ contacts and for $\mathrm{Cr}-\mathrm{Cr}$ oxide-Ag multilayered structures that, as we hope, may shed light on the possible role of inelastic scatterings of a magnetic origin at the injector-oxide interfaces. As is shown in the inset in Fig.2,b, on a large scale the conductance $\sigma(V)$ of the LBCO-based junction exhibits a quasi-linear behavior (2) with a superimposed superconducting gap-like feature. We relate it to chemical composition changes in the near-interface region that are usually attributed to oxygen
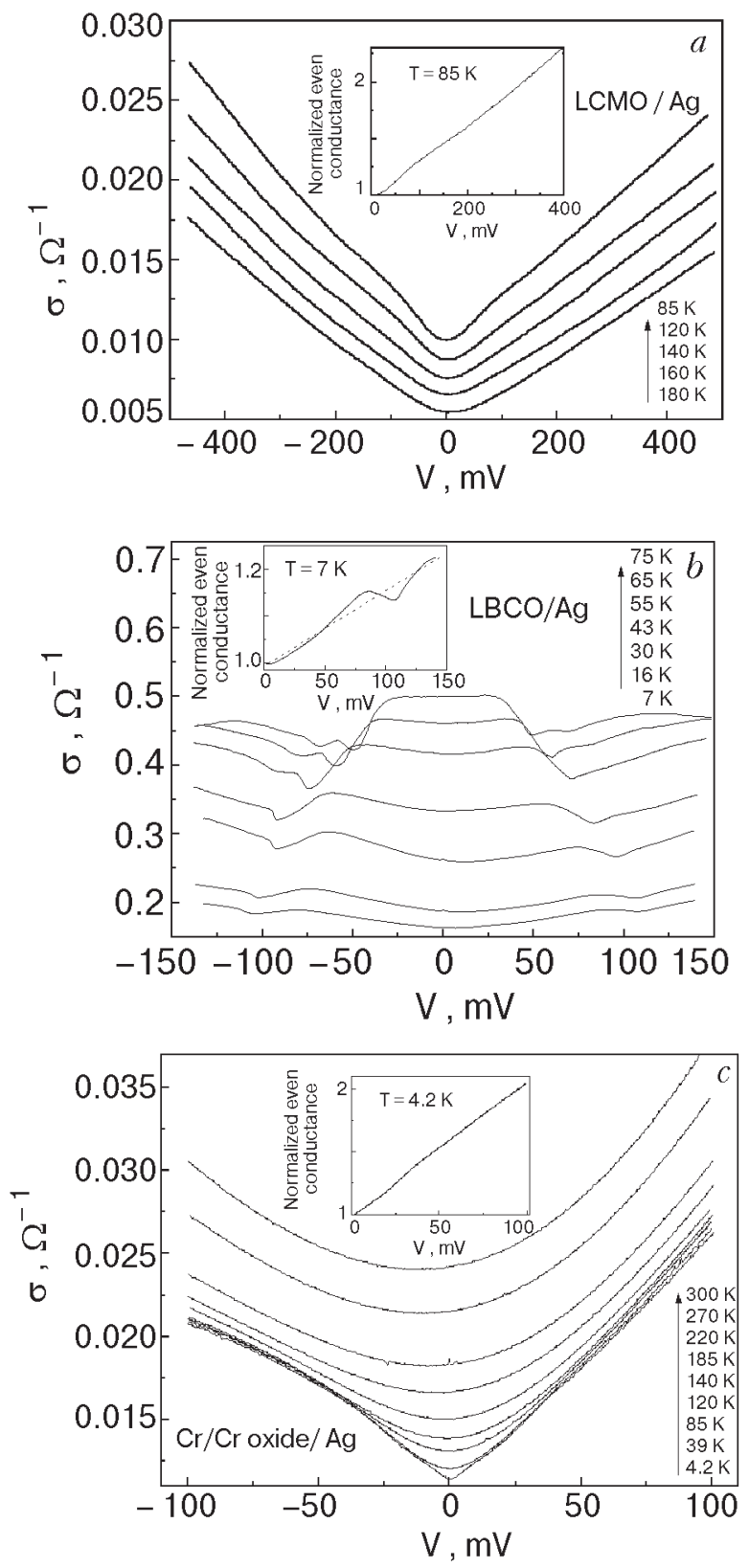

Fig.2. Temperature effect on the tunneling conductance spectra for a $\mathrm{La}_{0.66} \mathrm{Ca}_{0.34} \mathrm{MnO}_{3} / \mathrm{Ag}$ point contact (a), a $\mathrm{LaBa}_{2} \mathrm{Cu}_{3} \mathrm{O}_{7-x} / \mathrm{Ag}$ point contact (b), and a planar junction between $\mathrm{Cr}$ and $\mathrm{Ag}$ films with a magnetic chromium oxide as a potential barrier $(c)$. Insets show even parts of the differential conductance vs voltage curves at lowest measured temperatures. Note the dominating «V»-shaped background in all three characteristics.

ion displacement processes at the cuprate surface, leading, in particular, to strong suppression of superconductivity in the upper layers (see the review [9]). The most important thing for our purposes is the correlation between the strength of electromigration processes in LBCO (compared with YBCO and LCMO) and the quasi-linear over- 
all behavior of the differential conductance, which is known to serve as a fingerprint of the inelastic-tunneling effect [7].

Next, we consider the validity of the assumption of an oxygen-deficient near-interface layer created after application of corresponding high biases to the metallic injector and the existence of a magnetically active interlayer between the two electrodes that strongly influences electron tunneling processes across the transition region. It is believed [7] that the layer appears because of the removal of oxygen in the upper cuprate layers, which modifies the doping of $\mathrm{CuO}_{2}$ planes and can thus lead to long-range antiferromagnetic correlations between localized $\mathrm{Cu}$ spins. An argument for such a supposition comes from the paper [7], where it was shown that the anomalous $b|V|$ contribution to the conductance spectra of a normal metal/YBCO junction vanishes near the expected Néel temperature $T_{N}$. If we are really dealing with a magnetic barrier, the same observation should be true for heterostructures with both perovskite oxides. For those with LBCO we did not expect the oxygen removal to be as great as in the $\mathrm{YBCO} / \mathrm{Pb}$ specimens studied in the work [7]. The reason is that $\mathrm{Pb}$ deposited on the metaloxide surface strongly extracts oxygen atoms, forming a $\mathrm{PbO}_{x}$ barrier, but this does not occur for noble metals such as $\mathrm{Au}$ or Ag, whose standard reduction potential is known to be higher than that for $\mathrm{Cu}^{2+}$. Hence, $T_{N}$ for a surface region of LBCO should be somewhat lower than that found before [7] for YBCO-based contacts with $\mathrm{Pb}$. The temperature effect on the linear slope of $\sigma_{e}(V)$, i.e., on the coefficient $b$, is shown in Fig.3 for both structures. As to electromigration effects, the basic behavior of $b(T)$ for LBCO devices is the same as in [7] for YBCO contacts, but the temperature values at which $b$ decays are considerably lower. It is important that $b(T)$ for manganite samples goes to zero with increasing $T$ as well (Fig.3).

Why do we interpret the anomalous (proportional to $|V|$ ) term in the conductance spectra as being a result of inelastic boson-assisted tunneling? The standard theory of tunneling phenomena in metal heterostructures that takes into account only elastic transmission and the energy dependence of the tunneling matrix element [4] predicts the quadratic behavior (1). There are several possible reasons why a term proportional to $|V|$ can appear in $\sigma(V)$ : the non-Fermi-liquid nature of the oxides (the RVB model, the marginal Fermi-liquid hypothesis, etc.) as well as extrinsic approaches [10]. Following the paper [7], we reject effects of an intrinsic nature for superconducting cuprates (be-

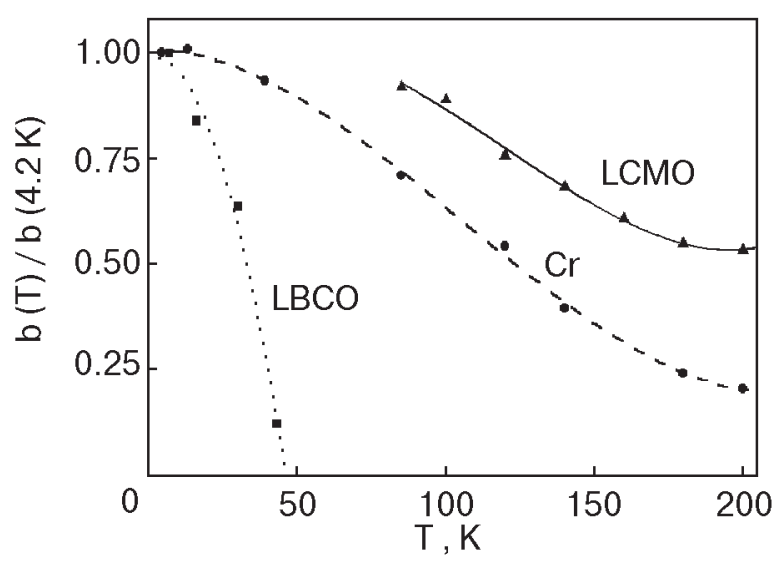

Fig.3. Temperature behavior of the parameter $b$ characterizing the strength of the inelastic tunneling processes for LCMO and LBCO based point contacts compared with the data for a $\mathrm{Cr}-\mathrm{Cr}_{2} \mathrm{O}_{3}-\mathrm{Ag}$ junction.

cause of the lack of any correlations between the temperature effect on the superconducting parameters and the coefficient $b$ ). The corresponding contribution to the even conductance $\sigma_{e}(V)$ can appear as a result of inelastic processes [11]. It is equal to $\int_{0}^{e V} F(\omega) d \omega$ and hence should be a linear function of the bias $V$ for a near-constant density of bosonic states $F(\omega)$ (in the case of magnetic excitations $F(\omega)$ is the dynamic susceptibility integrated over the wave vector). That is precisely the case for a normal YBCO compound [12] or an underdoped high- $T_{c}$ cuprate $\mathrm{La}_{2-x} \mathrm{Sr}_{x} \mathrm{CuO}_{4}$ [13], where magnetic inelastic neutron-scattering data reveal spin fluctuations in a large energy scale. We suppose that the broad continuum of these excitations is the origin of the unconventional linear term in $\sigma_{e}(V)$. Small nonlinearities in $\sigma_{e}(V)$ for LMCO and Cr oxide at voltages below $100 \mathrm{meV}$ (the insets in Figs. 2, $a$ and 2,c) reflect fine structures in the corresponding $F(\omega)$ [11].

The last argument for our statement about the magnetic nature of the enormous enhancement of inelastic tunneling processes is provided by a direct experiment with a junction in which an insulating interlayer between metallic electrodes is known to be magnetically ordered. For this purpose we have fabricated planar heterostructures between $\mathrm{Cr}$ and Ag thin films with a chromium oxide as a potential barrier. Such junctions were studied thirty years ago by Rochlin and Hansma [14], who showed that under appropriate oxidation conditions an antiferromagnetic $\mathrm{Cr}_{2} \mathrm{O}_{3}$ layer grows on the $\mathrm{Cr}$ film. At low temperatures, in accordance with our data for LCMO and LBCO, they observed a nearly 
linear dependence of $\sigma_{e}(V)$ on $V$, with a fine structure that was related to phonon and/or magnon excitations in the $\mathrm{Cr}_{2} \mathrm{O}_{3}$ barrier. Comparing these findings with those for similar Al-oxide junctions, the authors [14] concluded that the only significant difference between two sets of experiments was the magnetic behavior of $\mathrm{Cr}_{2} \mathrm{O}_{3}$, which must be responsible for increasing the inelastic tunneling contribution to the conductance of $\mathrm{Cr}-\mathrm{Cr}_{2} \mathrm{O}_{3}-$ metal heterostructures. We have followed the preparation procedure described by Rochlin and Hansma [14] and reproduced their data for $4.2 \mathrm{~K}$. Here we present detailed temperature measurements of $\mathrm{Cr}-\mathrm{Cr}$ oxide-Ag junction characteristics with a special emphasis on the anomalous $|V|$ term in $\sigma(V)$, regarded by us as an indication of inelastic tunneling via magnetic excitations in the oxide layer. As can be seen from Fig.2,c, this contribution is sufficiently strong and determines the overall behavior of the conductance background. We have plotted the strength of the process, i.e., the value of the coefficient $b$ versus temperature and compared it with analogous curves for perovskite-based junctions. From Fig.3 one can see a similar behavior of $b(T)$ for all three kinds of heterostructures. The abrupt decrease of the parameter $b$ with increasing temperature can be ascribed to the weakness of the spin fluctuations.

To summarize, we have performed conductance-versus-voltage measurements involving both cuprates and manganites, which we believe are important for deepening our understanding of the charge transport in heterostructures formed by these rather enigmatic materials. In the systems studied a degraded surface layer with enhanced magnetic correlations can play the role of a potential barrier, and in this case spin-assisted inelastic tunneling should strongly contribute to the charge tunneling transmission. To demonstrate this with independent experiments, we have fabricated planar junctions between $\mathrm{Cr}$ and $\mathrm{Ag}$ films with an insulating $\mathrm{Cr}_{2} \mathrm{O}_{3}$ layer formed by oxidizing the $\mathrm{Cr}$ film. This oxide in the bulk state is known to be antiferromagnetic at low temperatures. Despite different materials, barriers and preparation methods, the conductance spectra were found to be very similar, with an unusual $|V|$ term in the background dominating at low temperatures. Clearly, the only common feature of the samples studied is a magnetic interface between two metals forming the junction, and it is this that we suppose is responsible for the anomalous experimental findings. The obvious similarities between the three different sys- tems are not surprising because the underlying physics behind the observed phenomena is expected to be the same - an inelastic charge transmission across magnetically active insulating layers.

\section{Acknowledgements}

We thank Prof. M. A. Obolenskii, Prof. A. N. Omelyanchouk, and Prof. Yu. V. Medvedev for stimulating discussions. VMS is grateful to the Ministry of Education, Culture, Science and Technology of Japan for support of his stay at Hokkaido University and to the Research and Educational Fund of the Frostburg State University for support of his stay in the USA.

1. F. Guinea, Phys, Rev. B58, 9212 (1998).

2. P. LeClair, J. T. Kohlhepp, H. J. M. Swagten, and W. J. M. de Jonge, Phys. Rev. Lett. 86, 1066 (2001).

3. E. Hatta, V. M. Svistunov, Yu. F. Revenko, M. A. Belogolovskii, N. A. Doroshenko, and G. Plitnik, Modern Phys. Lett. B14, 819 (2000).

4. E.L.Wolf, Principles of Electron Tunneling Spectroscopy, Oxford University Press, New York (1985).

5. M. Belogolovskii, M. Grajcar, and P. Seidel, Phys. Rev. B61, 3259 (2000).

6. L. F. Rybalchenko, V. V. Fisun, N. L. Bobrov, I. K. Yanson, A. V. Bondarenko, M. A. Obolenskii, Fiz. Nizk. Temp. 17, 202 (1991); [Sov. J. Low Temp. Phys. 17, 105 (1991)]; L. F. Rybalchenko, I. K. Yanson, R. Borowski, and D. Wohlleben, Physica C224, 38 (1994).

7. A. Plecenik, M. Grajcar, P. Seidel, S. Takacs, A. Matthes, M. Zuzcak, and S. Benacka, Physica C301, 234 (1998).

8. Z. Y. Chen, A. Biswas, I. Zutic, T. Wu, S. B. Ogale, R. L. Greene, and T. Venkatesan, Phys.Rev. B, in press.

9. M. Yu. Kupriyanov and K. K. Likharev, Sov. Phys. Usp. 33, 340 (1990).

10. T. Hasegawa, H. Ikuta, K. Kitazawa, in: Physical Properties of High Temperature Superconductors III, D. M. Ginsberg (ed.) World Scientific Publ., Singapore (1992).

11. V. M. Svistunov, M. A. Belogolovskii, and A. I. Khachaturov, Phys. Usp. 36, 65 (1993).

12. P. Bourges, in: The Gap Symmetry and Fluctuations in High Temperature Superconductors, Plenum Press (1998)

13. H. Hiraka, Y. Endoh, M. Fujita, Y. S. Lee, J. Kulda, A. Ivanov, and R. J. Birgeneau, cond-mat/0104087 (2001).

14. G. I. Rochlin and P. K. Hansma, Phys. Rev. B2, 1460 (1970). 